\title{
Effects of Transcutaneous Electrical Acupoint Stimulation on Postoperative Cognitive Decline in Elderly Patients: A Pilot Study
}

\author{
Tianlin Liu ${ }^{1,2}$ \\ Chunping Yin' \\ Yanan $\mathrm{Li}^{\mathrm{I}}$ \\ Fang Gao' \\ Lili Yu ${ }^{1,2}$ \\ Zhigang Wang' \\ Qiujun Wang (D) \\ 'Department of Anesthesiology, The \\ Third Hospital of Hebei Medical \\ University, Shijiazhuang City, Hebei, \\ People's Republic of China; ${ }^{2}$ Department \\ of Anesthesiology, Cangzhou Central \\ Hospital, Cangzhou, Hebei, People's \\ Republic of China
}

Background: Postoperative cognitive decline (POCD) in the old ages seriously delays the rapid recovery. Here, we aimed to investigate the effects of transcutaneous electrical acupoint stimulation (TEAS) against POCD in elderly patients undergoing laparoscopic radical colon cancer surgery, as well as the potential mechanism.

Methods: A prospective, single-center, parallel-group, randomized trial was designed. A total of 100 patients (age $\geq 65$ years) undergoing laparoscopic radical resection of colon cancer were involved and randomly divided into TEAS (Group T) and control (Group C) groups. The patients in Group T were performed with percutaneous acupoint electrical stimulation in bilateral Hegu, Neiguan and Zusanli points from 30 minutes before anesthesia induction to the end of surgery. A Z-score based on Mini-Mental State Exam (MMSE) was used to assess the incidence of POCD. The levels of serum IL-6, hs-CRP, CGRP at $0 \mathrm{~min}$ before TEAS (T0), $1 \mathrm{~h}$ after beginning of surgery (T1) and the end of surgery (T2) were evaluated.

Results: Our data showed that the cumulative duration of POCD on postoperative day 2 and 3 in Group T was significantly decreased compared to Group $\mathrm{C}(P<0.05)$. Compared with T0, the levels of serum IL-6, hs-CRP, and CGRP in both Group T and C were statistically elevated at T1 and T2 $(P<0.05)$. Moreover, the levels of serum IL-6 and hs-CRP were decreased, but the level of CGRP was increased in Group T compared to Group C at T1 and T2 $(P<0.05)$.

Conclusion: TEAS is associated with a lower cumulative duration of POCD in elderly patients undergoing laparoscopic radical colon cancer surgery, which may be related to the regulation of inflammatory factors and neuropeptides interacted with gut-brain axis.

Keywords: cognitive decline, gut-brain axis, transcutaneous electrical acupoint stimulation, colon cancer, elder

\section{Introduction}

Postoperative cognitive decline (POCD) is usually defined as a significant alteration of cognitive function after anesthesia and surgery. As one of the common postoperative complications in elderly patients, POCD seriously affects the rapid recovery after surgery. In addition, it was reported that POCD also prolongs hospital stays and increases the long-term mortality in the population of old ages. ${ }^{1}$ However, the mechanism of POCD remains unclear. Until now, there are few efficient precautions and interventions against POCD.

It has been suggested that the inflammatory response to anesthesia and surgery may be involved into the incidence of POCD. ${ }^{2-5}$ Note that intestinal microbiota
Correspondence: Qiujun Wang

Tel +8631I 88602072

Email wangqiujunsy@I63.com 
perturbation-induced inflammatory response after surgical trauma has been revealed to play an important role in the pathogenesis and progression of cognitive dysfunction. ${ }^{6}$ The gut-brain axis, which is formed by the central nervous system, the enteric innervation that includes extrinsic fibers of the autonomous nervous system and intrinsic neurons of the enteric nervous system, the HPA-axis and the intestinal microbiota, may provide a fresh perspective between inflammatory response and subsequent cognitive decline. ${ }^{7}$ Moreover, the changes of intestinal microbiota via gut-brain axis are likely to be associated with the pathologies of Alzheimer's disease. ${ }^{8}$ In patients undergoing gastroenteric surgeries, increased levels of inflammatory factors released from injured intestinal tissue after anesthesia and surgery may be related to POCD. ${ }^{9}$ Thus, the inhibition of inflammatory response via gut-brain axis may become a potential therapy for cognitive dysfunction.

As a traditional Chinese medicine treatment, acupuncture has attached great importance to prevent occurrences and development of diseases. ${ }^{10}$ Compared with acupuncture alone, transcutaneous electrical acupoint stimulation (TEAS) using electrical stimulation on the skin surface exerts significant advantages, such as non-trauma, and acceptant. It was suggested that TEAS not only regulates the levels of neuropeptides, ${ }^{11}$ but also participates in the changes of gastrointestinal inflammatory factors. ${ }^{12}$ The antiinflammation mechanism of acupuncture may be related to the homeostatic restoration of intestinal microbiota. ${ }^{13}$ Previous studies from our group showed that TEAS can improve cognitive decline in elderly patients with focal lacunar infarction. ${ }^{14}$ However, the effects of TEAS on POCD in elderly patients undergoing laparoscopic radical resection of colon cancer remain to be explored.

In this current study, we aimed to investigate whether TEAS could reduce the incidence and duration of POCD in elderly patients undergoing laparoscopic radical resection of colon cancer. In addition, we determined the correlation between TEAS and gut-brain axis-associated inflammatory factors and neuropeptides.

\section{Methods}

\section{Patients and Setting}

The present study was performed with the approval of the ethics committee of Cangzhou Central Hospital (ethical register number: 2020-153-02) and was in compliance with the Helsinki Declaration. The project was registered in China Clinical Trial Registry (registration number:
ChiCTR2000040397). Written informed consents of the study were collected from all the candidate subjects before randomization. Patients who underwent laparoscopic radical resection of colon cancer in Cangzhou Central Hospital from December 1st, 2020 to February 15th, 2021 were enrolled into this trial. The inclusion criteria for patients who underwent surgery were as follows: (1) aged $\geq 65$ years; and (2) ASA grade I-II. The exclusion criteria included (1) history of neurological and psychiatric disorders; (2) use of anti-inflammatory drugs, glucocorticoids or other hormonal drugs, alcohol or drug dependence; (3) severe cardiac, pulmonary, hepatic, or renal dysfunction; (4) difficulty talking, aural or visual disorders; (5) skin damage located in the cutaneous acupoints performed; (6) the Mini-Mental State Exam (MMSE) $\leq 23$.

\section{TEAS}

According to a random number table, patients were randomly divided into two groups: control group (Group C) and TEAS group (Group T). The randomization was performed using an online randomization tool (http://www. randomization.com). From $30 \mathrm{~min}$ before the induction of anesthesia to the end of surgery, TEAS was performed in patients in Group $\mathrm{T}$ at acupoints Neiguan (PC6), Hegu (LI4) and Zusanli (ST36). TEAS was conducted under the guidance of acupuncturists who hold the post of attending physician in rehabilitation centers.

The acupoint Neiguan (PC6) is located on the palmar side of the forearm, two inches across the wrist, between the palmar longus tendon and the radial flexor carpi tendon. The acupoint Hegu (LI4) is located at the back of the hand, between the 1st and 2nd metacarpal bones, on the midpoint of the radial side of the 2 nd metacarpal bone. The location of Zusanli (ST36) is in the anterolateral leg, 3 inches under the acupoint Dubi, 1 transverse finger away from tibia anterior margin. Parameters were set as density wave, frequency $2-100 \mathrm{~Hz}$, and current intensity was the maximum patient could tolerate (Figure 1). In Group C, electrodes were placed in the same position as Group T, but no electric current stimulation was performed.

\section{Anesthesia Management}

Electrocardiograph (ECG), non-invasive blood pressure (NBP), pulse oxygen saturation $\left(\mathrm{SPO}_{2}\right)$, body temperature, end-tidal carbon dioxide partial pressure $\left(\mathrm{PetCO}_{2}\right)$ and bispectral index (BIS) were performed in all patients. Ultrasound-guided right internal jugular vein catheterization and right radial artery catheterization were performed 


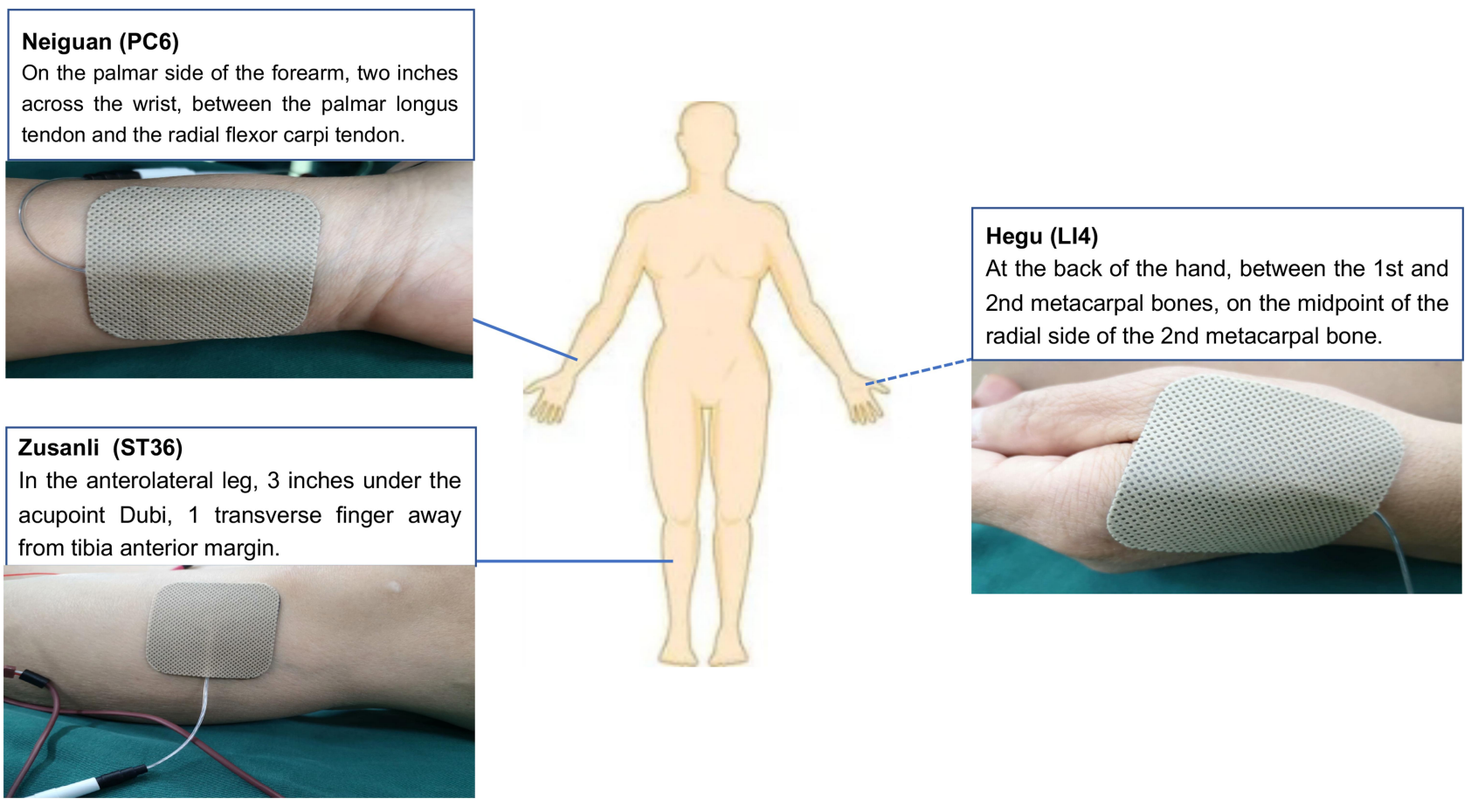

Figure I The location of the acupoints for transcutaneous electrical acupoint stimulation.

to continuously detect central venous pressure and invasive blood pressure.

\section{Anesthesia Induction}

Anesthesia was induced by intravenous injection of 2-5 $\mu \mathrm{g} / \mathrm{kg}$ fentanyl, $2-5 \mathrm{mg} / \mathrm{kg}$ propofol and $0.2-0.3 \mathrm{mg} / \mathrm{kg}$ cis-atracurium. 3 minutes later, tracheal intubation was performed using a video laryngoscope. The respiratory rate of ventilator was set to $12-20$ times per minute. The ratio of inspiratory/expiratory was 1.0:1.5, inhalation oxygen concentration was $40 \%$, oxygen flow was $2 \mathrm{~L} / \mathrm{min}$, and $\mathrm{PetCO}_{2}$ was maintained between 35 and $45 \mathrm{mmHg}$ (SL2400, Spacelabs, MA, UA).

\section{Maintenance of Anesthesia}

Anesthetic maintenance involved remifentanil (0.25-0.4 $\mu \mathrm{g} / \mathrm{kg} / \mathrm{min}$ ) plus propofol (total intravenous anesthesia [TIVA], $4-12 \mathrm{mg} / \mathrm{kg} / \mathrm{h}$ ) at the discretion of the anesthesiologist. The intravenous fluid goal was $500-$ to- $-1000 \mathrm{~mL} / \mathrm{h}$ for each hour. Cis-atracurium was intermittent intravenous injected to maintain muscle relaxation. BIS was maintained within the range of 40-60 (qCON2000, Quantium Medical Co Ltd, Spain). The fluctuation of MAP was kept within the range of $20 \%$ of the baseline. If mean arterial pressure (MAP) was lower or higher than $20 \%$ of the baseline, MAP changes were achieved by an infusion pump (CP-3100; Beijing Silugao Medical Technology Co Ltd, Beijing, China), which was used to administer norepinephrine or nicardipine (in $\mu \mathrm{g} / \mathrm{min}$ ), supplemented with boluses. If the patient represented significant bradycardia, atropine boluses $(0.3-0.5 \mathrm{mg})$ were administered. When patient's consciousness returns, coughing and swallowing reflexes return, tidal volume and ventilation per minute return to preoperative levels, tracheal intubation was removed.

\section{Postoperative Analgesia}

Electronic pump controlled intravenous analgesia (containing $0.15 \mu \mathrm{g} / \mathrm{kg}$ sufentanil in $100 \mathrm{~mL}$ saline) for all patients after surgery. The parameters were set as following: (flow rate, $2 \mathrm{~mL} / \mathrm{h}$; bolus, $0.5 \mathrm{~mL}$; lockout time, 15 min). Visual Analogue Score (VAS) was used to evaluate postoperative pain within $24 \mathrm{~h}$ after surgery, and the number of analgesic pump compressions was also recorded.

\section{Baseline Assessment}

Basic information including gender, age, height, weight, academic background (education $\geq 9$ years), physical status classification, and heavy drinking $(>40 \mathrm{~g} / \mathrm{d}$ for men or $>20 \mathrm{~g} /$ $\mathrm{d}$ for women) was collected. History of underlying diseases (hypertension, diabetes mellitus, coronary heart disease, hypoproteinemia), and smoking history were also collected. 


\section{Collection of Blood Samples}

As $0 \mathrm{~min}$ before TEAS (T0), $1 \mathrm{~h}$ after the beginning of surgery (T1), and the end of surgery (T2), $3 \mathrm{~mL}$ of blood was collected using an aseptic ethylene diamine tetraacetic acid tube. After centrifugation at 3,000 $\times \mathrm{g}$ for $15 \mathrm{~min}$, the collected serum was stored at $-80^{\circ} \mathrm{C}$. The levels of serum interleukin-6 (IL-6) (Lot: 131161131210, Code: EK0410, Boster Biological Technology Co, Wuhan, China), and calcitonin-gene-related peptide (CGRP) (Lot: 71515561210, Code: EK1169, Boster Biological Technology Co, Wuhan, China) were detected by ELISA according to the manufacturer's instructions. High sensitivity C-reactive protein (hsCRP) (Lot: 201110, Code: CH0402311, Maccura Biotechnology, China) was detected by latex immunoturbidimetric method according to the manufacturer's instruction.

\section{Assessment of POCD}

The Mini-Mental State Exam (MMSE) is the most often used short screening tool for providing a clear impression of overall cognitive decline and facilitating monitoring of the progress and resolution of delirium after surgery. ${ }^{15,16}$ MMSE score was assessed at one day before surgery, as well as postoperative days 1,2 , and 3 . The trained researchers who performed the MMSE score were blinded to the grouping of patients. As in previous publications, ${ }^{15,16}$ POCD was defined as a Z-score $\leq-2$ based on a pre- and postoperative MMSE. The following formula was used: [(postoperative MMSE-preoperative MMSE)- $\Delta \mathrm{X}$ MMSE normative population]/[SD ( $\Delta \mathrm{X}$ MMSE normative population)]. The $\triangle \mathrm{X}$ MMSE normative population is the mean value for changes in MMSE in a normative population and standard deviation $(\Delta \mathrm{X}$ MMSE normative population) is the standard deviation for changes in MMSE in a normative population, as measured in a cognitively normal sample. In this current study, $\Delta \mathrm{X}$ MMSE normative population $=0.5$, and $\mathrm{SD}(\Delta \mathrm{X}$ MMSE normative population) $=1.5$ were used to calculate Z-score. ${ }^{15}$ Once the patient developed POCD, an intramuscular injection of $5 \mathrm{mg}$ haloperidol was administered.

\section{Statistical Analyses}

MedCalc 15.2.2 software was used to calculate the sample size. According to previous reports, the incidence of POCD in the elderly population is $30 \%,{ }^{17}$ through calculation, the overall sample size was 94 . Considering a $10-20 \%$ abscission rate, a total of 105 patients were selected. All data were analyzed by SPSS (version 21.0 for Windows;
IBM Corporation, Armonk, NY, USA). The data were reported as the Mean $\pm \mathrm{SD}$ or Median \pm Quartile according to normal distribution. Categorical variables were analyzed through Chi-squared test or the Fisher's exact probability test (when appropriate) and presented as numbers (\%). Comparisons between two groups were performed with Student's $t$-test. Repeated measurements were compared using One-way repeated measures ANOVA, when the spherical hypothesis is not satisfied, GreenhouseGeisser $(P<0.75)$ and Huynh-Feldt $(P>0.75)$ method were used for correction. Tukey's multiple comparisons test was used for post-mortem analysis of one-way repeated measures ANOVA. The measurement data of non-normal distribution were tested by signed-rank test. $P<0.05$ was considered statistically significant.

\section{Results}

\section{Clinical Characteristics of Patients and Surgical Results}

During this study period, 105 patients who underwent laparoscopic radical resection of colon cancer were involved in this trial. Five patients were excluded because of history of neurological and psychiatric disorders $(\mathrm{n}=1$ [0.95\%]), use of anti-inflammatory drugs $(n=1[0.95 \%])$, aural disorders inability to cooperate $(\mathrm{n}=2[1.9 \%])$, skin damage located on the right ST36 acupoints $(\mathrm{n}=1$ [0.95\%]). The remaining 100 patients were randomly divided into Group T and Group C (Figure 2).

\section{Patient Characteristics}

The patients included into this final cohort were all Han Chinese, the general characteristics of the entire patients included gender [female, $49(49 \%)$ ], age $(70.24 \pm 5.143$ years), BMI $\left(24.78 \pm 3.04 \mathrm{~kg} / \mathrm{m}^{2}\right)$, American Society of Anesthesiologists (ASA) [52 (52\%)], education $\geq 9$ years [20 (20\%)], heavy drinking [19 (19\%)], smoker [49 (49\%)], preoperative hypoproteinemia [13 (13\%)], history of diabetes mellitus [25 (25\%)], hypertension [58 (58\%)], coronary heart disease [17 (17\%)], surgical duration (215.2 $\pm 55.623 \mathrm{~min})$, and anesthesia duration $(265.4 \pm 56.294 \mathrm{~min})$. The characteristics between patients in Group $\mathrm{T}$ and $\mathrm{C}$ are summarized in Table 1 (details in Supplementary Table S1).

\section{Intraoperative and Postoperative Variables}

There were no significant statistical differences of infusion volume, blood loss, urine volume, surgical duration and 


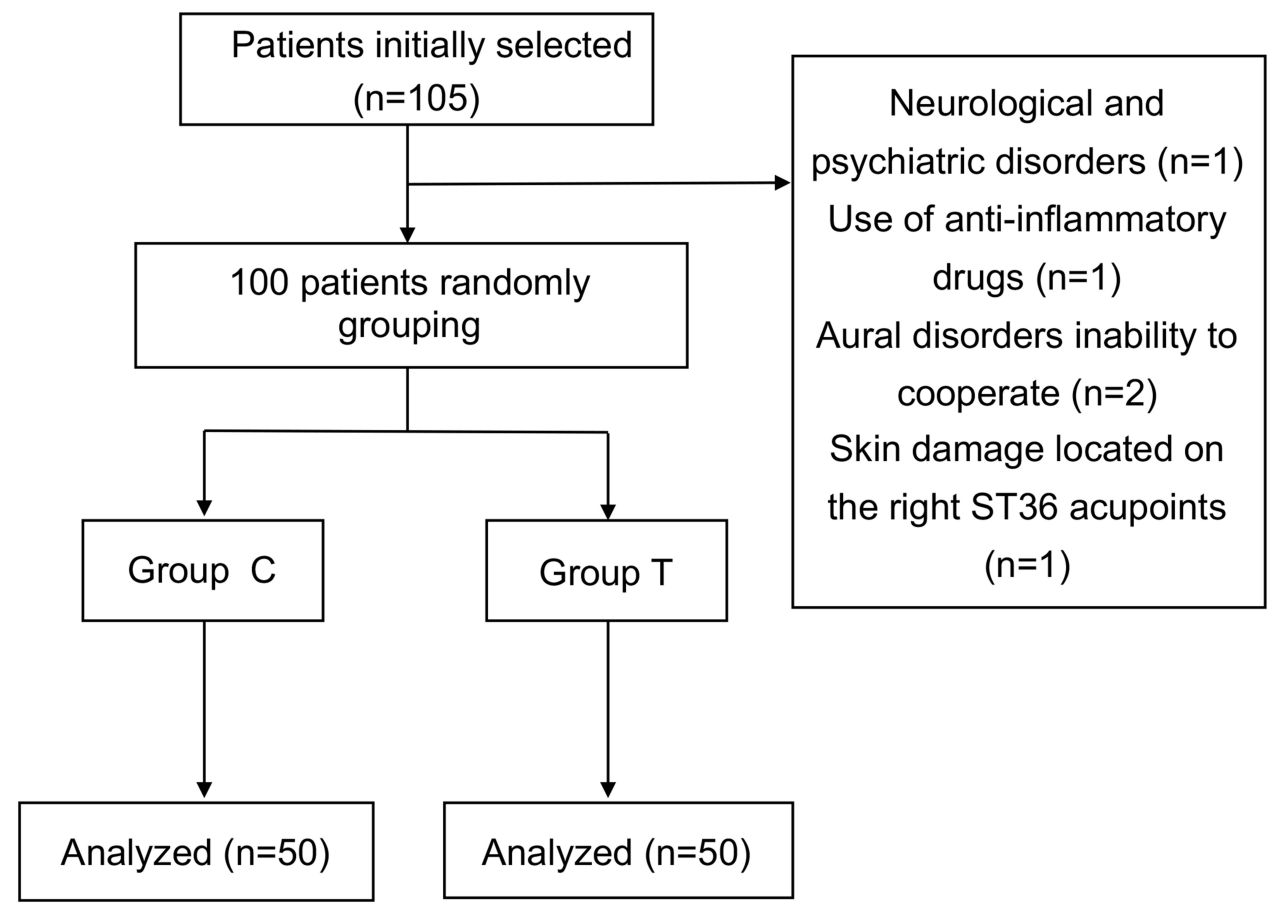

Figure 2 Experimental flow of this study.

anesthesia time between Group T and C (Table 2). For the postoperative variables, there were no differences in length of stays, VAS score and number of compressions $24 \mathrm{~h}$ after surgery between Group $\mathrm{T}$ and $\mathrm{C}$ (Table 2).

\section{IL-6, hs-CRP and CGRP Levels}

As shown in Figure 2, compared with T0, the levels of serum IL-6 $[\mathrm{F}(1.057,51.771)=415.596, P<0.001]$, hsCRP $[\mathrm{F}(1.584,77.602)=5075.619, P<0.001]$ and CGRP $[\mathrm{F}(1.715,84.039)=2107.192, P<0.001]$ were significantly increased at T1 and T2 in Group T. Likewise, the levels of serum IL-6 [F $(1.044,51.165)=615.190$, $P<0.001]$, hs-CRP $[\mathrm{F}(1.821,89.218)=5320.781, P<$ 0.001] and CGRP $[\mathrm{F}(1.776,87.013)=716.741, P<$ 0.001 ] were significantly increased at $\mathrm{T} 1$ and $\mathrm{T} 2$ in Group C. However, the levels of serum IL-6 $(\mathrm{t}=19.033$, $P<0.001$ for $\mathrm{T} 1 ; \mathrm{t}=22.733, P<0.001$ for $\mathrm{T} 2)$ and hsCRP $(\mathrm{t}=34.785, P<0.001$ for T1; $\mathrm{t}=23.906, P<0.001$ for T2) were decreased, but the level of CGRP $(\mathrm{t}=-8.290$, $P<0.001$ for $\mathrm{T} 1 ; \mathrm{t}=-22.652, P<0.001$ for $\mathrm{T} 2)$ was increased in Group T compared to Group C at T1 and T2 (Figure 3) (details in Supplementary Table S2).

Table I Clinical Characteristics of Patients

\begin{tabular}{|c|c|c|c|c|}
\hline Characteristics & Entire $(n=100)$ & Group T $(n=50)$ & Group $C(n=50)$ & $\mathbf{P}$ \\
\hline Age [years, mean (SD)] & $70.24(5.14)$ & $70.80(5.41)$ & $69.68(4.85)$ & 0.209 \\
\hline Gender [Female (\%)] & $49(49)$ & $22(44)$ & $27(54)$ & 0.317 \\
\hline Weight $[\mathrm{kg}$, mean (SD)] & $66.72(9.17)$ & $66.72(8.5 \mathrm{I})$ & $66.72(9.87)$ & 0.204 \\
\hline BMI $\left[\mathrm{kg} / \mathrm{m}^{2}\right.$, mean $\left.(\mathrm{SD})\right]$ & $24.78(3.04)$ & $24.75(2.57)$ & $24.82(3.47)$ & 0.114 \\
\hline ASA $(I, \%)$ & $52(52)$ & $25(50)$ & $27(54)$ & 0.689 \\
\hline Education $\geq 9$ years $[\mathrm{n}(\%)]$ & $20(20)$ & II (22) & $9(18)$ & 0.617 \\
\hline Hypertension [n (\%)] & $58(58)$ & $30(60)$ & $28(56)$ & 0.685 \\
\hline Diabetes mellitus [n (\%)] & $25(25)$ & II (22) & $14(28)$ & 0.488 \\
\hline Coronary heart disease $[\mathrm{n}(\%)]$ & $17(17)$ & $9(18)$ & $8(16)$ & 0.790 \\
\hline Hypoproteinemia [n (\%)] & $13(13)$ & $7(14)$ & $6(12)$ & 0.766 \\
\hline Smoker [n (\%)] & $49(49)$ & $24(48)$ & $25(50)$ & 0.841 \\
\hline Heavy drinking [n (\%)] & $19(19)$ & II (22) & $8(16)$ & 0.444 \\
\hline
\end{tabular}

Abbreviations: BMI, body mass index; ASA, American Society of Anesthesiologists; C, control; T, transcutaneous electrical acupoint stimulation. 
Table 2 Intraoperative and Postoperative Variables

\begin{tabular}{|c|c|c|c|c|}
\hline Variables & Entire $(n=100)$ & Group $T(n=50)$ & Group C $(n=50)$ & $\mathbf{P}$ \\
\hline Infusion volume [mL, mean (SD)] & $2121 \pm 521$ & $2189 \pm 498$ & $2053 \pm 539$ & 0.193 \\
\hline Blood loss [mL, mean (SD)] & $309 \pm 85$ & $305 \pm 85$ & $312 \pm 85$ & 0.683 \\
\hline Urine volume $[\mathrm{mL}$, mean $(\mathrm{SD})]$ & $442 \pm 112$ & $454 \pm 118$ & $430 \pm 105$ & 0.291 \\
\hline Surgical duration [minutes, mean (SD)] & $215.2 \pm 55.62$ & $225.6 \pm 53.28$ & $204.8 \pm 57.05$ & 0.063 \\
\hline Anesthesia time [minutes, mean (SD)] & $265.4 \pm 56.29$ & $272.2 \pm 56.88$ & $258.6 \pm 55.44$ & 0.229 \\
\hline VAS rest [point, mean (SD)] & $2.59 \pm 0.57$ & $2.52 \pm 0.505$ & $2.66 \pm 0.626$ & 0.221 \\
\hline VAS coughing [point, mean (SD)] & $3.18 \pm 0.63$ & $3.20 \pm 0.571$ & $3.16 \pm 0.68 \mid$ & 0.751 \\
\hline Number of compressions [times, mean (SD)] & $17.5 \pm 3.28$ & $17.62 \pm 2.687$ & $17.38 \pm 3.801$ & 0.716 \\
\hline Length of stays [days, mean (SD)] & $16.28 \pm 2.18$ & $16.10 \pm 2.30$ & $16.46 \pm 2.06$ & 0.412 \\
\hline
\end{tabular}

Abbreviations: C, control; T, transcutaneous electrical acupoint stimulation; VAS, Visual Analogue Scale.

\section{The Incidence of POCD}

On postoperative 1 (POD1), 5 cases $(10 \%)$ of POCD occurred in Group T, but 11 cases (25\%) in Group C. On POD2, 4 cases of POCD in Group T (8\%) but 8 cases in Group C (16\%) remain. On POD3, only 2 cases of POCD in Group T (4\%) but 5 cases in Group C (10\%) remain. In addition, there were no new cases on POD2 and 3. On POD1-3, the incidence of POCD was compared between the two groups was not statistically significant $(\mathrm{P}=0.102$, POD1; $\mathrm{P}=0.218$, POD2; $\mathrm{P}=0.436$, POD3). However, the cumulative duration of POCD was also used to evaluate the effects of TEAS. Interestingly, the cumulative duration of POCD on POD2 in Group T was significantly lower than Group $C(P=0.026)$. In addition, the cumulative incidence of POCD on POD3 in Group T was significantly lower than that in group $\mathrm{C}(\mathrm{P}=0.006)$ (Figure 4).

\section{Discussion}

In the present study, we found that TEAS significantly decreased the cumulative duration of POCD in old-aged patients receiving laparoscopic radical colon cancer surgery. In addition, inflammatory factors including IL- 6 and hs-CRP were heavily attenuated, but neuropeptides including CGRP was elevated after treatment with TEAS. The changes of inflammatory factors and neuropeptides may provide ideas for the effect of TEAS on the gut-brain axis.

The incidence of POCD in elderly patients undergoing laparoscopic radical resection of colon cancer has been reported to be $8.2-54 \% .{ }^{18}$ In the current study, we reported the incidence of POCD in the population received laparoscopic radical resection of colon cancer was $35 \%$, which consistent with a previous study. ${ }^{18}$ There are growing evidence that the risk factors of POCD include advanced age, surgical damages, anesthetic drug use, gut-brain axis imbalance and so on. ${ }^{19}$ In the elderly patients received radical resection of colon cancer, surgical damages, destruction of gut-brain axis, and cerebral degeneration may be involved in the incidence of POCD.

According to traditional Chinese medicine theory, Hegu is the original point of the large intestine meridian, the total treatment of the head and face of various diseases. Neiguan is one of the most important points in the body, which exhibits an excellent effect on neurological and mental system diseases. Besides, Neiguan is also one of the main points of the treatment for gastrointestinal
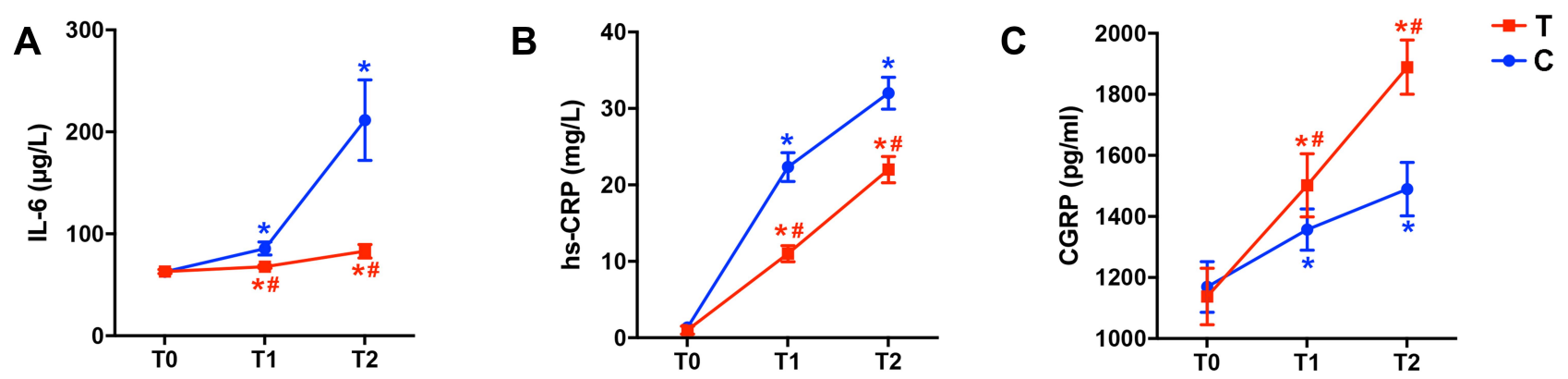

Figure 3 The level of IL-6 (A), hs-CRP (B), and CGRP (C) at different time points. Compared to T0, $* P<0.05$ in the same group. Compared to the corresponding Group C, ${ }^{\#} P<0.05$. T0: 0 min before transcutaneous electrical acupoint stimulation, TI: I h after the beginning of surgery; T2: the end of surgery.

Abbreviations: IL-6, interleukin-6; hs-CRP, high sensitive C-reactive protein; CGRP, calcitonin-gene-related peptide. 


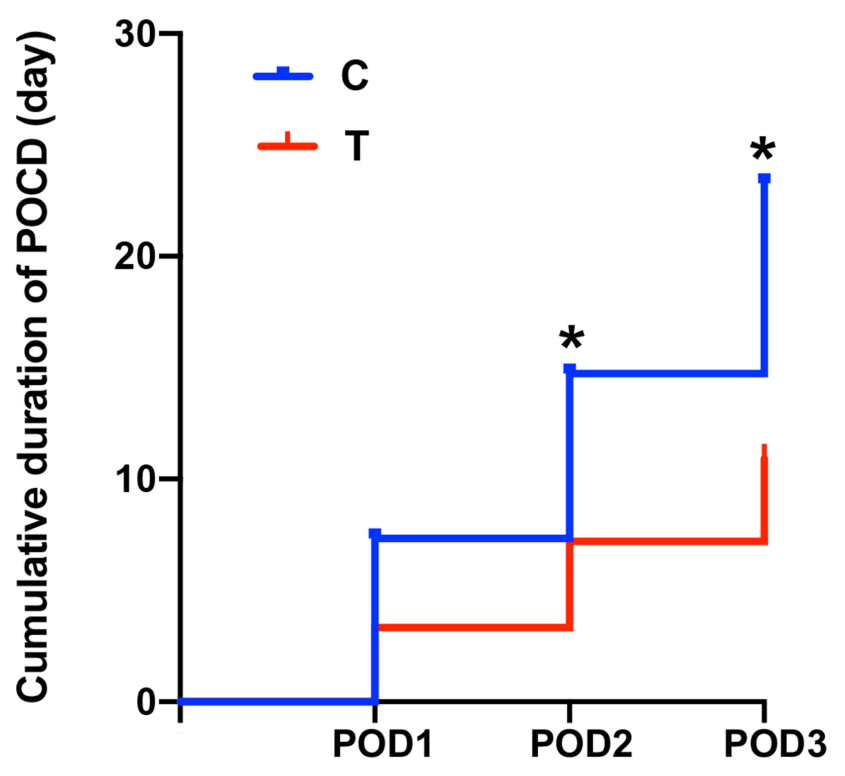

Figure 4 Cumulative duration of postoperative cognitive decline. Compared to the corresponding Group C, ${ }^{*} P<0.05$.

diseases. Zusanli is one of the main acupoints of "Zusanli stomach meridian". The stimulation of Zusanli may contribute to gastrointestinal peristalsis, appetizing, and digestion. Moreover, the stimulation of Zusanli has been also suggested to promote the recovery of cerebral dysfunction. ${ }^{20}$ Collectively, we selected these above acupoints that may contribute to bi-directional regulation of gut-brain axis. Although the incidence of POCD within the first 3 days after surgery in patients received TEAS was not significantly attenuated, but the cumulative duration of POCD on POD2 and 3 was significantly decreased compared to control, which is consistent with previous studies. $^{14,21}$

To further explore the roles of neuroinflammation in the pathogenesis of POCD, the levels of IL-6, hs-CRP and CGRP were assessed according to previous studies. ${ }^{5,17,22,23}$ IL-6, a key pro-inflammatory factor in the acute stage of trauma, is produced rapidly in response to infection and tissue damage. ${ }^{24} \mathrm{Hs}$-CRP, one of the most common markers of systemic inflammation, can rise sharply within a few hours once our body suffered from damage, inflammation, and infection. Some publications demonstrated that the concentration of serum hs-CRP is an independent risk factor of POCD in elderly patients undergoing laparoscopic colon cancer surgery. ${ }^{25}$ CGRP, one of the common neuropeptides distributed in the central nervous system, exhibits neuroprotective effects against ischemia/reperfusion injury and inflammatory responses. ${ }^{26,27}$ It was also suggested that
CGRP can improve learning and memory ability via the inhibition of tumor necrosis factor- $\alpha$ and induction of insulin-like growth factor $1 .^{28}$ In addition, CGRP have been demonstrated to play an important role in the communication of the gut-brain axis. ${ }^{29}$ Data from this study indicated that TEAS can reduce the levels of serum IL- 6 and hs-CRP to inhibit inflammatory response in the elderly during surgery, which is consistent with previous studies. ${ }^{14,21,30}$ Also, our data showed that the level of serum CGRP after treatments with TEAS was significantly elevated compared to control. These results revealed that the decreased cumulative duration of POCD after treatments with TEAS may be related to the changes of gut-brain axis-associated inflammatory factors and neuropeptides.

Admittedly, there were several limitations in the current study. Firstly, the number of patients was relatively small. Secondly, there were only three acupoints selected in the current study, and the other acupoints should be further investigated. Thirdly, in this current study, a MMSE was only used to evaluate the incidence of POCD, but more examinations should be applied. In addition, the long-term effects of TEAS on the incidence of POCD should be examined in our further study. Finally, we did not measure the length of the bowel resection, which might affect the results. However, despite these limitations, our results provided preliminary evidences of a neuroprotective effect of TEAS in patients undergoing intestinal surgery.

In conclusion, treatment with TEAS reduced the cumulative duration of POCD in patients undergoing laparoscopic radical colon cancer surgery. The mechanism may be related to the regulation of gut-brain axis-associated inflammatory factors and neuropeptides.

\section{Data Sharing Statement}

The datasets generated during and/or analyzed during the current study will be available upon reasonable request from Cangzhou Central Hospital. Email: 1377689587@qq. com; Telephone: +86 18003370383 .

\section{Ethical Approval}

The study was approved by Cangzhou Central Hospital Ethics Committee (ethical register number: 2020-153-02). Trial Registration: ChiCTR2000040397.

\section{Acknowledgments}

We would like to thank Limin Zhang, Zhaohui Liu and Yangyang Guo for their support of this project. This project was supported by the National Natural Science 
Foundation of China (81771134), Hebei Province technology Innovation guide Project Science and Technology Winter Olympics special project (19977790D), Hebei Provincial government funded the specialty capacity building and specialty leader training program.

\section{Author Contributions}

All authors made substantial contributions to the conception and design, acquisition of data, or analysis and interpretation of data; took part in drafting the article or revising it critically for important intellectual content; agreed to submit to the current journal; gave final approval of the version to be published; and agree to be accountable for all aspects of the work.

\section{Disclosure}

The authors report no conflicts of interest in this work.

\section{References}

1. Sun Y, Feng H, Zou T, et al. Assessment of risk factors for postoperative cognitive dysfunction after coronary artery bypass surgery: a single-centre retrospective cohort study. Biosci Rep. 2021;41. doi:10.1042/BSR20190719

2. Faraco G, Brea D, Garcia-Bonilla L, et al. Dietary salt promotes neurovascular and cognitive dysfunction through a gut-initiated TH17 response. Nat Neurosci. 2018;21(2):240-249. doi:10.1038/ s41593-017-0059-Z

3. Ho YS, Zhao FY, Yeung WF, et al. Application of acupuncture to attenuate immune responses and oxidative stress in postoperative cognitive dysfunction: what do we know so far? Oxid Med Cell Longev. 2020;2020:9641904. doi:10.1155/2020/9641904

4. Glumac S, Kardum G, Karanovic N. Postoperative cognitive decline after cardiac surgery: a narrative review of current knowledge in 2019. Med Sci Monit. 2019;25:3262-3270. doi:10.12659/ MSM.914435

5. Glumac S, Kardum G, Sodic L, et al. Effects of dexamethasone on early cognitive decline after cardiac surgery: a randomised controlled trial. Eur J Anaesthesiol. 2017;34(11):776-784. doi:10.1097/EJA.000 0000000000647

6. Yang XD, Wang LK, Wu HY, et al. Effects of prebiotic galacto-oligosaccharide on postoperative cognitive dysfunction and neuroinflammation through targeting of the gut-brain axis. BMC Anesthesiol. 2018;18(1):177. doi:10.1186/s12871-018-0642-1

7. Agustí A, García-Pardo MP, López-Almela I, et al. Interplay between the gut-brain axis, obesity and cognitive function. Front Neurosci. 2018;12:155. doi:10.3389/fnins.2018.00155

8. Lin C, Zhao S, Zhu Y, et al. Microbiota-gut-brain axis and toll-like receptors in Alzheimer's disease. Comput Struct Biotechnol J. 2019;17:1309-1317. doi:10.1016/j.csbj.2019.09.008

9. Wang R, Chen J, Wu G. Variable lung protective mechanical ventilation decreases incidence of postoperative delirium and cognitive dysfunction during open abdominal surgery. Int J Clin Exp Med. 2015;8(11):21208-21214.

10. Cheng FK. The use of acupuncture in patients with Parkinson's disease. Geriatr Nurs. 2017;38(4):302-314. doi:10.1016/j. gerinurse.2016.11.010
11. Kaptchuk TJ. Acupuncture: theory, efficacy, and practice. Ann Intern Med. 2002;136(5):374-383. doi:10.7326/0003-4819-136-5-2002030 $50-00010$

12. Bai YF, Gao C, Li WJ, et al. Transcutaneous electrical acupuncture stimulation (TEAS) for gastrointestinal dysfunction in adults undergoing abdominal surgery: study protocol for a prospective randomized controlled trial. Trials. 2020;21(1):617. doi:10.1186/s13063-02004470-4

13. Jang JH, Yeom MJ, Ahn S, et al. Acupuncture inhibits neuroinflammation and gut microbial dysbiosis in a mouse model of Parkinson's disease. Brain Behav Immun. 2020;89:641-655. doi:10.1016/j. bbi.2020.08.015

14. Gao F, Zhang Q, Li Y, et al. Transcutaneous electrical acupoint stimulation for prevention of postoperative delirium in geriatric patients with silent lacunar infarction: a preliminary study. Clin Interv Aging. 2018;13:2127-2134. doi:10.2147/CIA.S183698

15. Hensel A, Angermeyer MC, Riedel-Heller SG. Measuring cognitive change in older adults: reliable change indices for the mini-mental state examination. J Neurol Neurosurg Psychiatry. 2007;78 (12):1298-1303. doi:10.1136/jnnp.2006.109074

16. Momeni M, Meyer S, Docquier MA, et al. Predicting postoperative delirium and postoperative cognitive decline with combined intraoperative electroencephalogram monitoring and cerebral near-infrared spectroscopy in patients undergoing cardiac interventions. J Clin Monit Comput. 2019;33(6):999-1009. doi:10.1007/s10877-019-00253-8

17. Quan C, Chen J, Luo Y, et al. BIS-guided deep anesthesia decreases short-term postoperative cognitive dysfunction and peripheral inflammation in elderly patients undergoing abdominal surgery. Brain Behav. 2019;9(4):e01238. doi:10.1002/brb3.1238

18. Scholz AF, Oldroyd C, McCarthy K, et al. Systematic review and meta-analysis of risk factors for postoperative delirium among older patients undergoing gastrointestinal surgery. Br J Surg. 2016;103(2): e21-28. doi:10.1002/bjs.10062

19. Lin X, Chen Y, Zhang P, et al. The potential mechanism of postoperative cognitive dysfunction in older people. Exp Gerontol. 2020;130:110791. doi:10.1016/j.exger.2019.110791

20. Tida JA, Catalão CHR, Garcia CAB, et al. Acupuncture at ST36 exerts neuroprotective effects via inhibition of reactive astrogliosis in infantile rats with hydrocephalus. Acupunct Med. 2018;36 (6):386-393. doi:10.1136/acupmed-2017-011515

21. Zhang Q, Li YN, Guo YY, et al. Effects of preconditioning of electro-acupuncture on postoperative cognitive dysfunction in elderly: a prospective, randomized, controlled trial. Medicine. 2017;96(26):e7375. doi:10.1097/MD.0000000000007375

22. Safavynia SA, Goldstein PA. The role of neuroinflammation in postoperative cognitive dysfunction: moving from hypothesis to treatment. Front Psychiatr. 2018;9:752. doi:10.3389/ fpsyt.2018.00752

23. Lei D, Sha Y, Wen S, et al. Dexmedetomidine may reduce IL-6 level and the risk of postoperative cognitive dysfunction in patients after surgery: a meta-analysis. Dose-Response. 2020;18(1):1559325820902345. doi:10.1177/1559325820902345

24. Tanaka T, Narazaki M, Kishimoto T. IL-6 in inflammation, immunity, and disease. Cold Spring Harb Perspect Biol. 2014;6(10):a016295. doi:10.1101/cshperspect.a016295

25. Xiang D, Xing H, Tai H, et al. Preoperative C-reactive protein as a risk factor for postoperative delirium in elderly patients undergoing laparoscopic surgery for colon carcinoma. Biomed Res Int. 2017;2017:5635640. doi:10.1155/2017/5635640

26. Edvinsson L, Haanes KA, Warfvinge K, et al. CGRP as the target of new migraine therapies - successful translation from bench to clinic. Nat Rev Neurol. 2018;14(6):338-350. doi:10.1038/s41582-018-0003-1

27. Tian J, Yang L, Wang P, et al. Exogenous CGRP regulates apoptosis and autophagy to alleviate traumatic brain injury through Akt/mTOR signalling pathway. Neurochem Res. 2020;45(12):2926-2938. doi:10.1007/s11064-020-03141-9 
28. Yang X, Shi Z, Li X, et al. Impacts of stellate ganglion block on plasma NF- $\mathrm{kB}$ and inflammatory factors of TBI patients. Int $J$ Clin Exp Med. 2015;8(9):15630-15638.

29. Wei P, Keller C, Li L. Neuropeptides in gut-brain axis and their influence on host immunity and stress. Comput Struct Biotechnol J. 2020;18:843-851. doi:10.1016/j.csbj.2020.02.018
30. Chen Y, Huang W, Li Z, et al. The effect of acupuncture on the expression of inflammatory factors TNF- $\alpha$, IL-6,IL-1 and CRP in cerebral infarction: a protocol of systematic review and metaanalysis. Medicine. 2019;98(24):e15408. doi:10.1097/MD.0000 000000015408

\section{Publish your work in this journal}

Clinical Interventions in Aging is an international, peer-reviewed journal focusing on evidence-based reports on the value or lack thereof of treatments intended to prevent or delay the onset of maladaptive correlates of aging in human beings. This journal is indexed on PubMed Central, MedLine, CAS, Scopus and the Elsevie
Bibliographic databases. The manuscript management system is completely online and includes a very quick and fair peer-review system, which is all easy to use. Visit http://www.dovepress.com/ testimonials.php to read real quotes from published authors.

Submit your manuscript here: https://www.dovepress.com/clinical-interventions-in-aging-journal 